\title{
Análisis del servicio de salud en la provincia de Santa Fe a la luz de los principios de equidad y eficiencia espacial
}

\section{MARÍA MERCEDES CARDOSO*, GRISELDA CARÑEL ** NORMA BEATRIZ FINELLI**}

*Facultad de Humanidades y Ciencias, Universidad Nacional del Litoral, Argentina; CONICET, Argentina.

** Facultad de Humanidades y Ciencias, Universidad Nacional del Litoral, Argentina. mercecardoso@hotmail.com; griseldacargnel@gmail.com; finellinormab@gmail.com;

\section{RESUMEN}

El grado de cobertura y acceso al servicio de salud es indicativo de diferencias en el desarrollo territorial. Los 55 municipios que conforman el área de estudio son representativos de las distintas regiones de la provincia de Santa Fe. Con el objetivo de realizar un diagnóstico territorial del sistema de salud, se recurre a un tratamiento cuantitativo de datos y al análisis a través del Sistema de Información Geográfica (SIG). La distribución espacial de los diferentes parámetros del sistema de salud presenta notorias desigualdades regionales, poniendo en cuestión el grado de ajuste de este servicio público asistencial a los principios de eficiencia y equidad socio-espacial. Como contribución para la gestión de políticas públicas, se identifica una concentración de efectores de salud y áreas de mayor accesibilidad a los centros de salud en los municipios más poblados de los departamentos de Rosario y La Capital, mientras que, en otros menos poblados, se evidencian importantes carencias.

PALABRAS CLAVE: servicio de salud; accesibilidad; eficiencia espacial; equidad socio-espacial; provincia de Santa Fe. 


\section{ABSTRACT}

ANALYSIS OF HEALTH SERVICE DATA IN SANTA FE PROVINCE IN TERMS OF SPATIAL EFFICIENCY AND EQUITY

The degree of coverage and accessibility to a health service in a certain territory signals differences in its development. The area under study comprises 55 municipalities which are representative of the different regions in Santa $\mathrm{Fe}$ province. Aiming at providing a territorial diagnosis of the health system, a quantitative treatment of data and a SIG analysis are carried out. The spatial distribution of different health system parameters exposes noticeable regional inequalities, and raises the question of the degree of adjustment of this care public service to the principles of efficiency and socio-spatial equity. In the most populated municipalities of Rosario and La Capital a concentration of health centers and greater accessibility areas to them have been identified, whereas in smaller locations important shortages of availability are revealed. This analysis can contribute to the management of public policies.

KEYWORDS: health service; accessibility; spatial efficiency, socio-spatial equity; Santa Fe province 


\section{RESUMO}

\section{ANÁLISE DO SERVIÇO DE SAÚDE NA PROVÍNCIA DE SANTA FE BASEADA NOS PRINCÍPIOS DE EQUIDADE E EFICIÊNCIA ESPACIAL}

O grau de cobertura e o acesso ao serviço de saúde são sinais das diferenças no desenvolvimento territorial. A área de estudo abrange os 55 municípios representativos das diferentes regióes da província de Santa Fe. Com o intuito de fazer um diagnóstico territorial do sistema de saúde, procede-se ao tratamento quantitativo de dados e à análise através do Sistema de Informação Geográfica-SIG- Com base nos princípios de eficiência e equidade socioespaciais, observa-se que a distribuição no território provincial dos diferentes parâmetros do sistema de saúde, apresenta marcadas desigualdades regionais, colocando-se no alvo de análise o grau de ajuste deste serviço de assistência pública. De modo a contribuir na gestão de políticas públicas, verifica-se uma maior concentração de centros de atendimento de saúde e áreas com maior acessibilidade nos municípios mais populosos, que integram os Departamentos de Rosario e La Capital; náo obstante, nas localidades com menor população evidenciam-se importantes carências destes serviços.

PALAVRAS-CHAVE: SERVIÇO de saúde; acessibilidade; eficiência espaciais; equidade socioespaciais; província de Santa Fe. 


\section{Introducción}

Según el último diagnóstico de la Comisión Económica para América Latina y el Caribe (CEPAL) sobre el panorama social de América Latina, los estados latinoamericanos asignan mayores recursos a nivel de gobierno central, a la protección social, a la educación y a la salud que a otro tipo de sectores (como vivienda, y otros servicios comunitarios). En 20I6, como promedio de los países de América Latina, "los gastos destinados a estas funciones representaron el 4,I\%, el 3,9\% y el 2,2\% del PIB, respectivamente" (CEPAL, 2019: 23). Chile y Uruguay son los países que disponen de más recursos por persona para políticas sociales (2.387 y 2.25I dólares, respectivamente), seguidos del Brasil (I.63I dólares), la Argentina (I.469 dólares) y Costa Rica (I.I76 dólares). En contraste, El Salvador y Bolivia alcanzan promedios de alrededor de 3io dólares y Guatemala, Nicaragua y Honduras de menos de 220 dólares.

En el concierto latinoamericano, si bien los recursos destinados a salud son unos de los que más crecieron en los últimos i6 ańos, las carencias y profundas desigualdades de acceso siguen siendo críticas, volviéndose una de las aristas más complicada de revertir dentro del flagelo de la pobreza. Después de $\mathrm{I} 2$ años en que las tasas de pobreza y pobreza extrema "se redujeron considerablemente, en 2015 y 2016 se registraron incrementos sucesivos de ambas tasas" (CEPAL, 2019: 20). Las cifras de 2017 muestran un incremento adicional de la pobreza extrema, mientras que la tasa de pobreza no presentó variaciones respecto del valor registrado en el año anterior. Para 2018 se espera que el crecimiento del PIB contribuya a una ligera reducción de la tasa de pobreza y una mantención de la tasa de pobreza extrema.

La salud representa tanto un derecho, como una necesidad de cada individuo para tener una vida digna, de calidad y que pueda contribuir, como sujeto, al desarrollo del conjunto de la sociedad. Para el estado, la salud comprende uno de los gastos significativos a los que debe hacer frente; la prestación de este servicio (como a todos los esenciales y asistenciales) y la garantía de acceso a toda la población implica una gestión eficiente de los recursos, de por sí escasos.

El presente trabajo se focaliza en las principales cualidades del servicio de salud en 55 municipios de la Provincia de Santa Fe, analizándose los rasgos de dicho servicio, del equipamiento, de su cobertura y distribución geográfica. El principal objetivo que orienta esta investigación 
es realizar un diagnóstico del estado actual del servicio de salud en el área de estudio y su grado de ajuste a los principios de eficiencia espacial y equidad socio-espacial. La metodología, en un primer momento, pone a consideración información del contexto provincial, recurriendo al análisis de datos aportados por informes oficiales de organismos de gobierno. Luego, a partir de la determinación de los parámetros de estudio y la generación de indicadores se lleva a cabo un tratamiento cuantitativo, basado en su análisis espacial a través de herramientas de los SIG y a su representación cartográfica. El producto representa un diagnóstico para la gestión de políticas públicas, contribuyendo al conocimiento de la distribución geográfica de esta oferta y demanda, así como de los posibles factores incidentes en la eficiencia de este servicio.

El trabajo analiza la temática descripta particularmente en los 55 municipios santafesinos, a fin de dar cuenta de las disparidades entre las diferentes regiones en que puede subdividirse la provincia. Justifica este estudio la necesidad de identificar los desequilibrios territoriales provinciales relativos a la salud, a fin de su dimensionamiento, para en ulteriores estudios, poder monitorear su evolución.

Los servicios básicos esenciales (agua, luz, saneamiento, recolección de basura, etc.), como los asistenciales (salud, seguridad, educación) (Antúnez y Galilea, 2003) persiguen el objetivo de la eficiencia y la equidad socio-espacial. Desde esta perspectiva contribuyen en términos generales al desarrollo.

La localización de los servicios resulta ser de gran importancia en muchos aspectos, especialmente en el ámbito de los servicios públicos, pues se orienta a la mejora de los niveles de calidad de vida y equidad espacial de la población.

El estudio de la relación entre la localización de los centros de salud y el comportamiento de ciertos indicadores como la proporción de población con NBI, pone en evidencia el cumplimiento o no de ese criterio de eficiencia y equidad. De manera indirecta, el análisis de la localización de los centros de salud estaría representando la oferta de ese servicio en particular, y la demanda satisfecha (de mejor o peor manera), más las áreas con accesibilidad a los centros públicos con internación general. Una medida de la eficiencia de la prestación del servicio de salud (sea público o privado) es el índice de pacientes en los principales centros poblados, donde se concentra la demanda. 
Este trabajo se enmarca en el Proyecto de investigación CAI+D UNL (Universidad Nacional del Litoral) "Calidad de vida en la Provincia de Santa Fe a inicios del siglo XXI. Aproximación a los factores de diferenciación”, iniciado en 2017.

\section{Metodología}

El área de estudio se compone por 55 municipios pertenecientes a la provincia de Santa Fe. Según la Ley Provincial 2756/86, un "distrito" (gobierno local) tiene una "municipalidad" encargada de la administración comunal cuando alcanza la cifra de Io.00o habitantes. La selección se realizó bajo criterios de integrar las diferentes cohortes poblaciones (los más poblados), desde Rosario y Santa Fe, primeros en la jerarquía urbana provincial, hasta aquellos que apenas sobrepasan los I0.000 habitantes -como Frontera, Las Parejas, Las Toscas- así como aquellos que no alcanzan la mencionada cifra -Sastre, Malabrigo, San Guillermo, entre otros-. Asimismo, los municipios elegidos cumplen con la condición de ser representativos de las distintas regiones geográficas de la provincia, tomando en consideración regionalizaciones como la de Manzi y Gallardo (1970).

Para el análisis se emplean los programas específicos de SIG: ArcGis I0.3 y QGis 2.18 Las Palmas y sus herramientas. Los SIG constituyen un instrumento de gran importancia en el monitoreo de los patrones espaciales de los fenómenos sociales, y en la visualización de problemas socioeconómicos. Sirven para la exploración de sectores de población en riesgo, de manera tal que si un SIG se mantiene en forma operativa y gerencial, puede agilizar los procesos de prevención e intervención institucional.

Se elaboró una matriz de datos, incorporando una serie de variables que posibilitan analizar algunos aspectos claves de la situación del sistema de salud y de las condiciones generales de la población que demanda dicho servicio. Las variables consideradas (población total en 2010 a escala de distrito, población con NBI, población con NBI tipo sanidad) se tomaron del Censo Nacional de Población, Hogares y Viviendas (Instituto Nacional de Estadística y Censos, INDEC, 20Io). Las variables relativas a la prestación del servicio de salud, cantidad de centros de salud de diferentes categorías, centros con internación general y vías de comunicación de acceso a los distritos que contienen esos centros, se 
obtuvo del Ministerio de Salud de la Provincia de Santa Fe y del Instituto Provincial de Estadística y Censo (IPEC).

\section{Contexto teórico-conceptual}

En la definición y clasificación de los servicios intervienen factores cruciales como el criterio tomado en cuenta. Es posible distinguir los servicios según el mercado al que van destinados; así, existen servicios a empresas o consumo intermedio o a personas, o consumo final; según la titularidad o propiedad, (servicios públicos y privados); el grado de modernidad (tradicionales y nuevos); la trascendencia en el desarrollo (motores/no motores; dirección/servicio); su frecuencia de consumo (banales/raros), etc. (Moreno Jiménez y Escolano Utrilla, 1992). En los estudios geográficos, la contraposición servicios públicos- servicios privados resulta de especial interés, principalmente en su distribución espacial. Los servicios privados o de mercado se comportan espacialmente atendiendo a las fuerzas de mercado, incluyendo factores relativos a la demanda, conducta de los competidores, etc. El árbitro de su nacimiento, localización, permanencia o desaparición es la rentabilidad económica. Los servicios públicos, más o menos sometidos al control administrativo, están más pendientes a criterios como la accesibilidad o disponibilidad para sus demandantes, que de la rentabilidad, de ahí derivan importantes implicaciones para su localización. La relevancia de este criterio radica en que, según se trate de uno o de otro, cabe esperar resultados territoriales distintos. No obstante, en la práctica, esta claridad en la distinción se desdibuja pues existen problemas derivados de cambios en la propiedad de los servicios (de privados pueden pasar a públicos o viceversa), o la interdependencia o interpenetración entre actividades públicas y privadas.

La salud, junto con la seguridad y la educación se consideran servicios urbanos asistenciales, diferentes de los esenciales, como pueden ser los vinculados a las condiciones de habitabilidad (luz eléctrica, agua de red, cloaca, espacios verdes, gas de red, etc.).

El rol de los servicios en el desarrollo económico y tecnológico de las regiones y países es crucial. Según Antúnez y Galilea (2003) los principales problemas que afectan a las ciudades latinoamericanas, con referencia a los servicios, remiten a un estado acumulado de déficit en su 
acceso y calidad, a situaciones de desigualdad e integración social y a complejas inercias e ineficacias en su gestión institucional.

En la organización del territorio se involucran una serie de principios que rigen la localización y distribución de los servicios básicos, entre ellos, la salud. Ellos son el principio de cohesión socio-territorial, eficiencia espacial y competitividad, calidad de vida y bienestar, sostenibilidad, equidad socio-espacial y justicia ambiental (Moreno Jiménez y Vinuesa Angulo, 2009).

En el caso de esta investigación interesa ahondar en los principios de eficiencia espacial-competitividad y equidad socio-espacial. En cuanto a la eficiencia espacial y su vinculación con la competitividad, refiere a la necesaria relación entre los recursos utilizados y los resultados obtenidos, es decir, partiendo de que los recursos económicos del estado (para los centros de salud públicos) o de los agentes económicos (caso de los privados) son escasos, este principio apunta a maximizar los logros en materia de cantidad de efectores y la calidad de la prestación. La eficiencia espacial difiere de la económica (que se mide por el beneficio en términos monetarios) pues considera otros aspectos como los resultados en término de confort-consenso social, mejoras ambientales, entorno cultural favorecedor de las relaciones sociales, de la vida democrática, en comunidad, etc.

Otro principio de interés es el de equidad socio-espacial, encaminado a evitar la discriminación y desigualdades entre individuos de distintos sectores. La equidad socio-espacial refiere a la noción de igualdad, principio rector de las ideas democráticas, que significa tratar igual a los iguales (individuos en similares circunstancias). Es relevante el criterio para el reparto social y espacial, y es en este punto en el que el principio se vincula a la justicia. La equidad socio-espacial en el uso y acceso al espacio constituye una premisa fundamental de toda política pública, para evitar la discriminación entre personas de diferentes lugares, con especial énfasis en los servicios urbanos asistenciales, que responden a necesidades vitales como la salud, el alimento, el abrigo. Ante la discusión respecto a cómo actuar con equidad en el reparto real de beneficios y perjuicios que se derivan del acceso a los servicios, se destacan dos aspectos a considerar: I- La igualdad espacial, cuyo contenido se compone de la igualdad de oportunidades y la igualdad de acceso; 2- La justicia territorial, que vaticina la provisión de recursos según las necesidades y, bajo el criterio rawlsiano, mejorar al máximo la situación de los menos favorecidos (Moreno Jiménez y Vinuesa Angulo, 2009). 
En el concepto de equidad se incluye la accesibilidad. La idea de accesibilidad geográfica encierra dos dimensiones distintas pero complementarias, la accesibilidad física y la accesibilidad socioeconómica. La accesibilidad física expresa la capacidad de la población para hacer uso de tal servicio e incluye la consideración de la aptitud del mismo, así como las condiciones o normas que regulan su funcionamiento. La accesibilidad socioeconómica alude a la existencia misma del servicio, así como a los medios de pago para llegar hasta él (Joseph y Phillips, 1984). La accesibilidad es un concepto multidimensional que incluye: a) la existencia (aparición y localización específica del servicio); b) la conectividad (red de circulación que permite su conexión a los puntos de demanda); c) la disponibilidad (posibilidad de absorber la demanda); e) la adecuación (la oferta cumple con las expectativas de la demanda) y f) la capacidad económica (medios de demanda para llegar al servicio) (Buzai y Baxendale, 2006).

En el presente trabajo se aborda la localización de los servicios y la conectividad de los mismos a través de la red vial, como un modo de aproximación al análisis de ciertos aspectos de la accesibilidad.

El acceso a los servicios genera externalidades (positivas o negativas) identificadas como los elementos o características del medio urbano que proporcionan bienestar y que contribuyen a la calidad de vida de las personas, familia o grupo allí residente -no pagados, ni cobrados-. Estos elementos generan efectos externos que no se reflejan directamente en los costos, ni en los precios. En toda ciudad existe una variación de las externalidades en el plano, ya que la distribución de bienes y de males en la ciudad no es uniforme. El tendido de la red y la calidad de los servicios públicos es uno de los principales factores de producción de externalidades. Se habla de "precios de accesibilidad" hacia las externalidades positivas y un "coste de proximidad" a las externalidades negativas (Harvey, 1973).

\section{Área de estudio}

El área de estudio delimitada en los 55 municipios (figura I) comprende 2.599.187 habitantes; representa el 81,3\% del total poblacional provincial (Censo Nacional de Población, Hogares y Viviendas, INDEC, 20Io). 


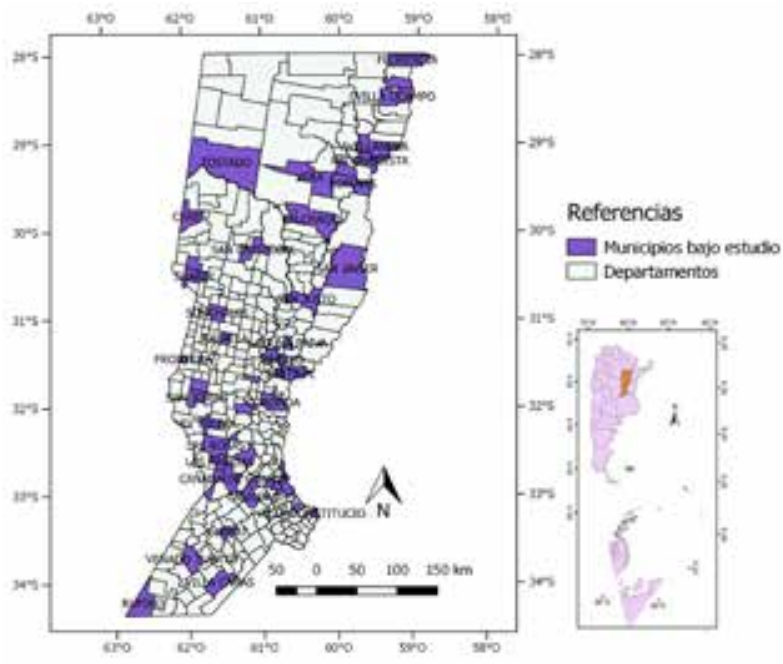

Figura 1. Localidades del área de estudio en la Provincia de Santa Fe

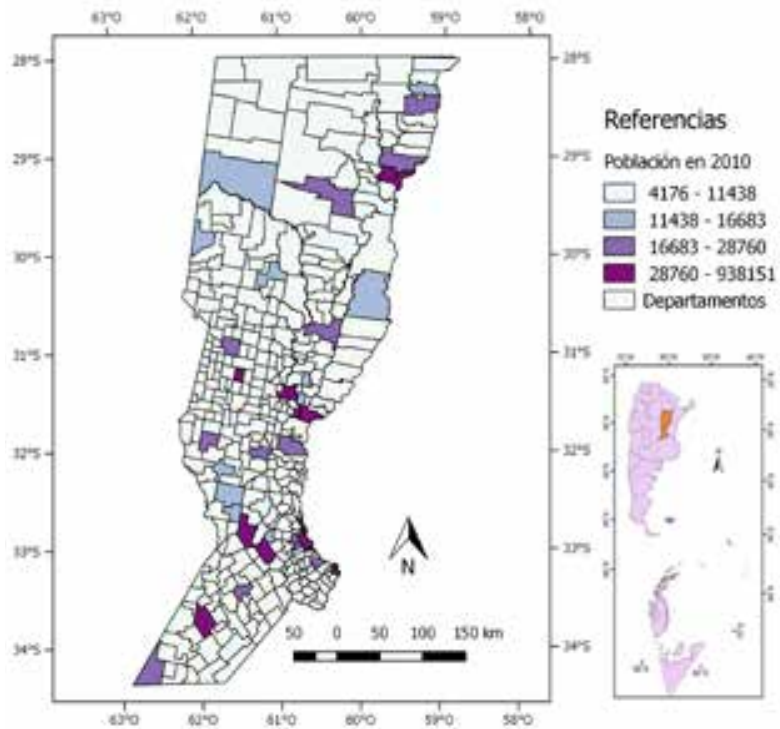

Figura 2. Localidades del área de estudio por categorías según montos poblacionales (2010)
Fuente: elaboración de las autoras en base a datos del IPEC (2010)

Fuente: elaboración de las autoras en base a datos del IPEC (2010) 
La distribución de la población (figura 2) presenta significativas desigualdades a nivel provincial, encontrándose mayores concentraciones demográficas en el sur (en torno al área metropolitana de Rosario y Venado Tuerto), en segundo lugar, en el centro (en torno a la capital provincia, área metropolitana de Santa $\mathrm{Fe}$ ) y demás ciudades medias y finalmente en el norte provincial donde, entre los distritos menos poblados, se destaca Reconquista, Avellaneda, un aglomerado de tamaño intermedio (cuadro I). Al interior de los distritos también pueden identificarse disparidades. En términos generales, en relación con la distribución poblacional, se distribuye la demanda de los servicios.

\section{Análisis y discusión ¿Quiénes demandan el servicio de salud?}

La población, en diferentes etapas de su vida, demanda servicios de salud en sus diversas modalidades y especialidades. Allí donde se concentra la población, se concentran demandas. En el sur-este provincial, en torno a Rosario y su área metropolitana se localiza el principal punto de demanda del servicio de salud. Constituye un aglomerado que supera el millón de habitantes, siendo la tercera ciudad en la jerarquía urbana nacional, y primera en la provincial. El segundo punto más poblado se ubica en el centro-este provincial, en el Gran Santa Fe, representando menos de la mitad que el primero. La tercera aglomeración en importancia también se ubica en el sur provincial (Venado Tuerto); en el escasamente poblado norte santafesino, el cuarto punto de demanda de servicios es el aglomerado Reconquista Avellaneda.

Cuadro 1. Principales distritos del área de estudio, cantidad de habitantes

\begin{tabular}{l|l}
\hline Distrito & Población en $\mathbf{2 0 1 0}$ \\
\hline Rosario & 938.151 \\
\hline Santa Fe & 386.860 \\
\hline Reconquista & 72.771 \\
\hline Venado Tuerto & 45.772 \\
\hline Rafaela & 92.945 \\
\hline Avellaneda & 25.906 \\
\hline
\end{tabular}

Fuente: elaboración propia a partir de CNPHyV, 2010. INDEC. 
La salud es un derecho y una necesidad básica de las personas. La figura 3 representa cartográficamente la distribución de la población con mayores necesidades, no solo del servicio de salud, sino de toda la batería de servicios esenciales y asistenciales. Allí se expresa el mapa de las carencias de la provincia de Santa Fe, ya que la variable valorada es población con necesidades básicas insatisfechas (en porcentajes), categorizados por quintiles, quedando en cada clase el $20 \%$ de las unidades espaciales.



Figura 3. Población con NBI en los municipios bajo estudio de la Provincia de Santa Fe, por categorías según porcentajes (2010)

Según el INDEC, se considewran Hogares con Necesidades Básicas Insatisfechas aquellos que presentan al menos una de las siguientes condiciones de privación: • NBI I. Vivienda: son los hogares que viven en habitaciones de inquilinato, hotel o pensión, viviendas no destinadas a fines habitacionales, viviendas precarias y otro tipo de vivienda. 
Se excluye a las viviendas tipo casa, departamento y rancho. - NBI 2. Condiciones sanitarias: incluye a los hogares que no poseen retrete. $\bullet$ NBI 3. Hacinamiento: es la relación entre la cantidad total de miembros del hogar y la cantidad de habitaciones de uso exclusivo del hogar. Operacionalmente se considera que existe hacinamiento crítico cuando en el hogar hay más de tres personas por cuarto. - NBI 4. Asistencia escolar: hogares que tienen al menos un niño en edad escolar ( 6 a I2 años) que no asiste a la escuela. - NBI 5 . Capacidad de subsistencia: incluye a los hogares que tienen cuatro o más personas por miembro ocupado y tienen un jefe que no ha completado el tercer grado de escolaridad primaria.

La figura 3 expresa los porcentajes de población con NBI categorizándolos en 5 clases, donde el verde oscuro corresponde al nivel bajo, el verde claro, al medio-bajo, el amarillo, a medio, el naranja al medioalto y el rojo al alto, representando la población con mayores carencias y condiciones de vida más desfavorables. Del análisis de dicha cartografía surge que la distribución de los diferentes sectores se da de la siguiente manera: los niveles bajos y medios bajos de NBI se localizan en el centro y sur provincial, señalando que las necesidades básicas de estas poblaciones tienen la cobertura más favorable dentro del concierto provincial. El nivel medio se encuentra tanto en distritos del norte, como del centro y sur provincial. El nivel medio-alto se ubica en más distritos del norte y en algunos del centro (Recreo y Coronda) y del sur (Pérez y Villa Gdor Gálvez, en el área de avance de Rosario). Por último, con los mayores porcentajes de población con NBI, en el norte provincial, Florencia representa la categoría en rojo, con casi el 30\% de su población con estas carencias. 


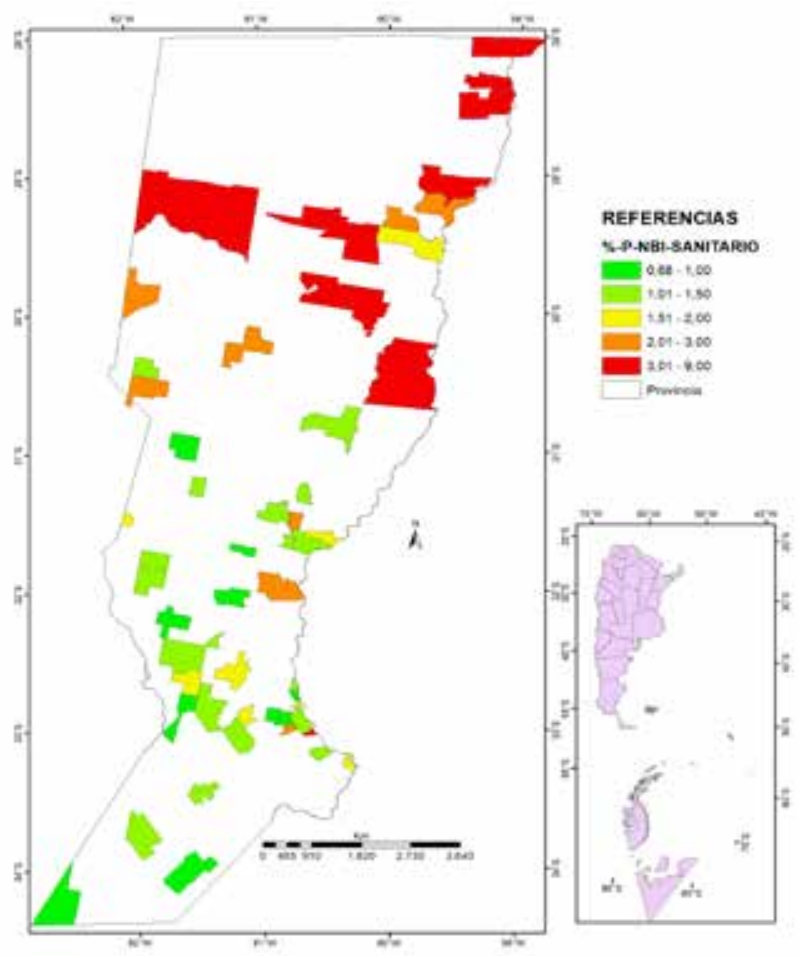

Fuente: elaboración de las autoras en base a datos del Censo Nacional de Población, Hogares y Viviendas (2010) IPEC.

Figura 4. Población con NBI tipo "sanitario" en los municipios bajo estudio de la Provincia de Santa Fe, por categorías según porcentajes (2010)

La figura 4 focaliza el diagnóstico de las condiciones de carencia de la población, a través de la distribución de los diferentes niveles de población con NBI sanitario, es decir, aquellos que no cuentan con retrete en su hogar, estando en una permanente situación de riesgo a su salud.

La distribución de los diferentes niveles de la variable en cuestión se presenta de esta manera: los bajos y medios bajo se ubican en los distritos del centro y sur provincial; los valores medios, en el norte, centro y sur; la categoría medio alto, en varios distritos del norte, nuevamente en Recreo y Coronda del centro provincial, y en Pérez, al sur. Los distritos en rojo, con los mayores valores de población con NBI sanitario se localizan mayoritariamente en el norte, son más que los de la figura 2, y uno del sur: Villa Gdor. Gálvez. 


\section{Análisis de la oferta del servicio de salud en Santa Fe}

El sistema público de salud tiene 783 centros de atención de diferentes jerarquías y jurisdicciones (provincial y municipal), de los cuales 437 se encuentran en el área de estudio (en 55 municipios). De estos, 299 son provinciales y 138 municipales.

La oferta del servicio de salud público (centros de atención primaria) en el área de estudio se representa en la figura 4, en su distribución puntual. Teniendo en cuenta que el interés del análisis espacial es reconocer concentraciones o dispersiones de los efectores de salud, se recurrió a la estrategia gráfica de los puntos de calor, como sitios de mayor concentración de centros.

Fuente: elaboración de las autoras en base a datos del Ministerio de Salud de la Provincia de Santa Fe (2017)

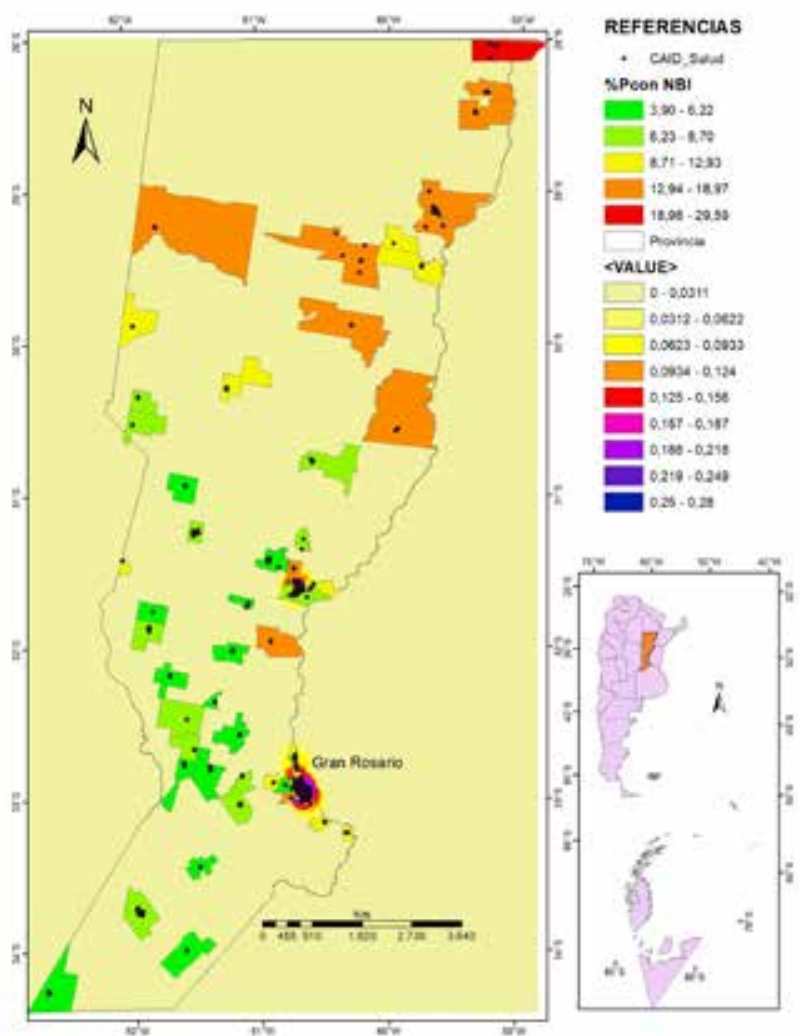

Figura 5. Concentración de centros de salud de distintas categorías en la Provincia de Santa Fe (2017) 
Los cinco puntos de calor corresponden a las máximas concentraciones de centros de salud: Rosario, Santa Fe, Rafaela, Reconquista-Avellaneda y Venado Tuerto.

Cuadro 2. Cantidad de Centros de Salud en los principales puntos de la Provincia de Santa Fe. Según categoría.

\begin{tabular}{l|l|l|l|l|l}
\hline Distrito/Categoría & \multicolumn{1}{l}{ Provincial } & \multicolumn{1}{l}{ Municipal } & \multicolumn{2}{l}{ Privado } & \multicolumn{2}{l}{ Pública } & \multicolumn{1}{c}{ Total } \\
\hline Rosario & 47 & 59 & 609 & 106 & $\mathbf{7 4 7}$ \\
\hline Santa Fe & 55 & 1 & 112 & 56 & $\mathbf{1 6 8}$ \\
\hline Rafaela & 12 & 0 & 17 & 12 & $\mathbf{2 9}$ \\
\hline Venado Tuerto & 3 & 13 & 31 & 16 & $\mathbf{4 9}$ \\
\hline Reconquista-Avellaneda & 24 & 0 & 4 & 24 & $\mathbf{2 8}$ \\
\hline Total & 141 & 73 & 773 & 214 & $\mathbf{1 0 2 1}$ \\
\hline
\end{tabular}

Fuente: Infraestructura de Datos Espaciales, Santa Fe. IDESF (2017)

El cuadro 2 presenta información detallada de lo expresado en los puntos de calor de la Figura 5. Se destaca la cantidad de centros de salud de Rosario: 747, la mitad de todos los centros de la provincia de Santa Fe. En un $82 \%$ son privados, y entre los públicos, en partes casi iguales son de gestión provincial y municipal.

A pesar de ser la capital provincial, el distrito Santa Fe, comparado con Rosario tiene un significativamente menor número de centros (I68), de los cuales son privados el $67 \%$ y de los públicos, exceptuando uno municipal, los demás con provinciales.

Venado Tuerto, con menor cantidad que el anterior, tiene semejantes proporción de centros privados y públicos, aunque se diferencia en la mayor presencia de los centros municipales ( 13 en total).

El último punto de calor, ubicado al norte provincial, muy distanciado de los anteriores, es el que aglomera a Reconquista y Avellaneda; en este caso de los 28 centros de salud existentes, 24 , es decir el $86 \%$ son públicos, de gestión provincial; solo 4 son privados. Estas cifras ponen de manifiesto un mayor énfasis puesto en la salud por parte de las políticas públicas.

Rafaela, con semejante cantidad de centros que Reconquista-Avellaneda, tiene levemente mayor proporción de privados que de públicos. 
Fuente: elaboración de las autoras en base a datos del Ministerio de Salud de la Provincia de Santa Fe (2017)



Figura 6. Concentración de centros de salud de distintas categorías en el aglomerado Gran Rosario, Provincia de Santa Fe (2017) 
Cuadro 3. Índice de pacientes. Número de habitantes por centros de salud, en principales puntos de oferta del servicio. Según categoría.

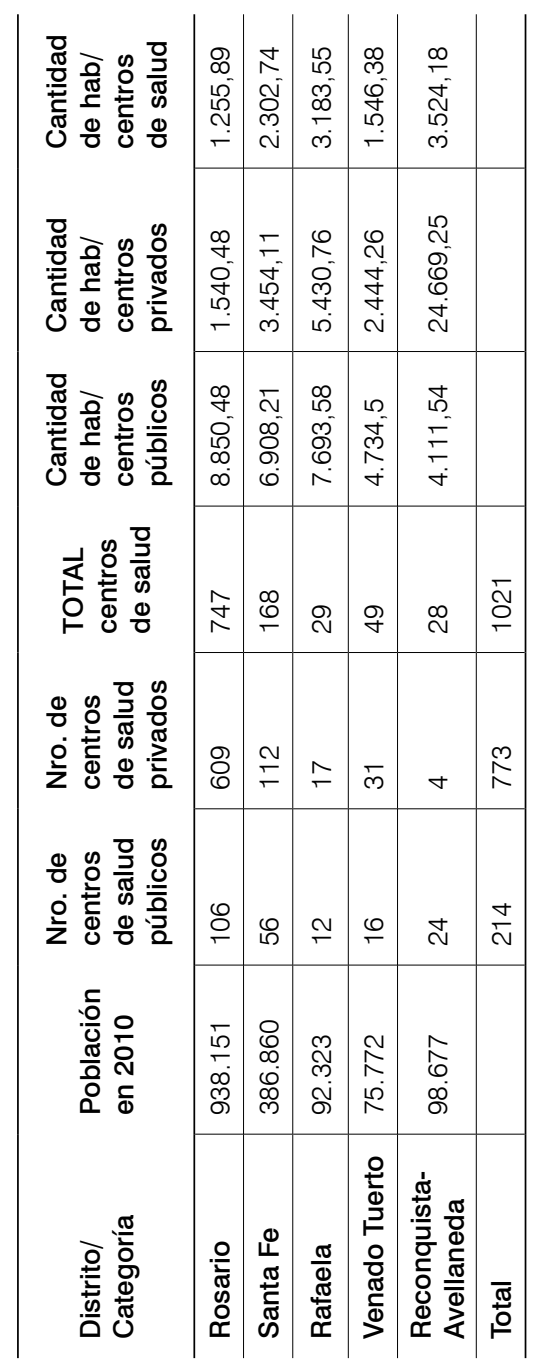

Fuente: Infraestructura de Datos Espaciales, Santa Fe. IDESF (2017) 
Los centros de salud privada compensan las necesidades de un sistema de salud pública saturada o colapsada para los casos de Rosario, Santa Fe y Venado Tuerto, haciendo disminuir la cantidad de habitantes por centros de salud en general. No resulta suficiente para los casos de Rafaela y Reconquista, cuyos valores de número de habitantes por centro de salud a atender pasan los 3.000. La situación es notoriamente grave para el caso del aglomerado del norte santafesino, donde los valores de población con NBI son los más altos de la provincia.

\section{Análisis de la accesibilidad al servicio de salud}

En la determinación de las áreas de influencia bruta de los hospitales (área de acceso por caminos y rutas en tiempos definidos) se calculó la isócrona de 60 minutos. Ésta se basó en la clasificación de los caminos según su tipo de calzada. A cada tipo de calzada se atribuyó una velocidad (cuadro 4) que permitió el cálculo del tiempo de recorrido en minutos de cada tramo.

Cuadro 4. Velocidad de desplazamiento de la red vial en el área de estudio

\begin{tabular}{|c|c|c|}
\hline TIPO & CLASE & VELOCIDAD $(\mathrm{Km} / \mathrm{hr})$ \\
\hline Autopista & Pavimentado & 130 \\
\hline Ruta & Pavimentado & 110 \\
\hline Camino & Pavimentado & 70 \\
\hline Camino & Consolidado & 50 \\
\hline Camino & Tierra & 40 \\
\hline Senda & Tierra & 20 \\
\hline
\end{tabular}

Fuente: elaboración propia en base a datos de Dirección Provincial de Vialidad, Santa Fe (2017)

En la elaboración de la figura 7 se utilizaron los siguientes datos: vectores de rutas nacionales y provinciales, y caminos vecinales, elaborados por la Dirección Provincial de Vialidad (2017); categorías y localización de los hospitales públicos provinciales según el Ministerio de Salud de la provincia de Santa Fe. Se llevó a cabo un análisis de redes de los caminos y los Hospitales con Internación General. Para ellos se 
tomó como nodos los hospitales y la red vial que llega a ellos. Las áreas de influencia se determinaron mediante la envolvente de 60 minutos de viaje desde el centro hospitalario en todas las direcciones a través de la red de rutas y caminos (Savoy et al. 2012).

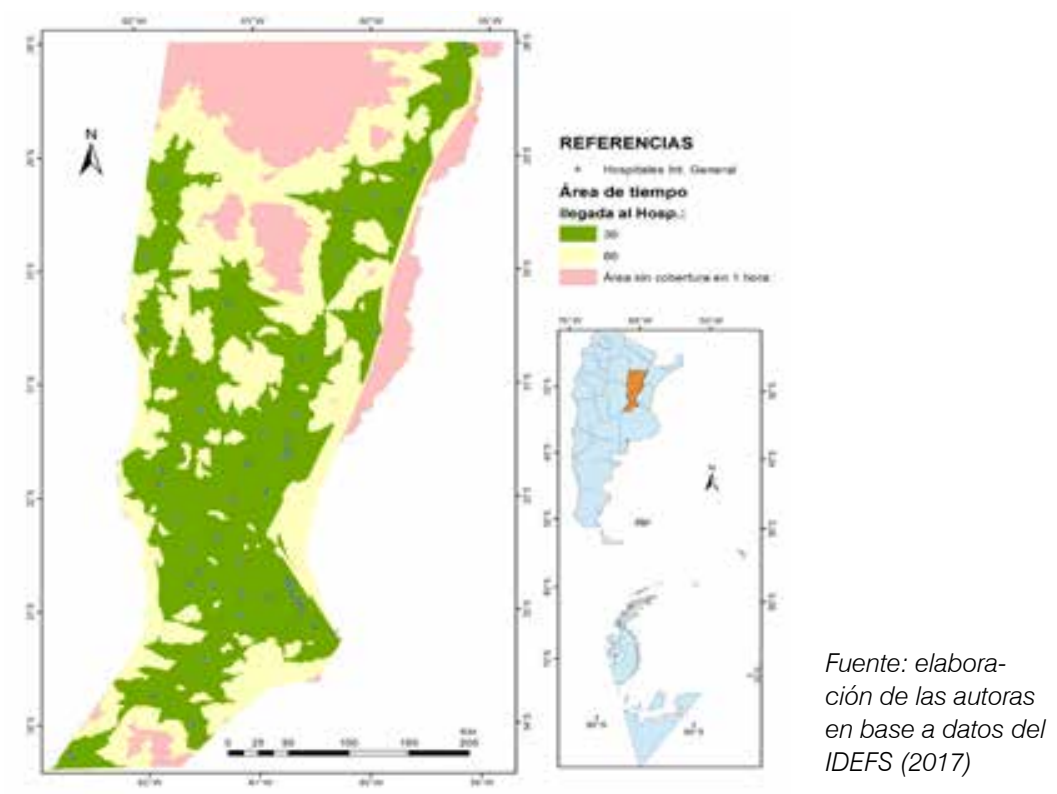

Figura 7. Accesibilidad en tiempo a los centros de Internación General (Hospitales públicos) en la Provincia de Santa Fe (2017)

En el territorio provincial quedan áreas de inaccesibilidad a los centros de internación público (a más de $\mathrm{I}$ hora), principalmente en el norte, y unas pequeñas porciones en el sur. Por otro lado, en torno al Gran Rosario (y en menor medida en Santa Fe), existe superposición de áreas de accesibilidad, denotando los desequilibrios. 


\section{Conclusiones}

Los servicios públicos en general, y el de salud en particular, deben estar especialmente pendientes de criterios como la accesibilidad, guiados por principios fundamentales como la eficiencia espacial y la equidad social. La cartografía producto de la investigación desarrollada muestra que en la provincia de Santa Fe existen grandes desequilibrios en la concentración de la población, la distribución de los centros urbanos en el territorio y la jerarquía urbana. A su vez, la población con mayores carencias se localiza preferentemente en el norte provincial, de gran extensión territorial (población con NBI).

En cuanto a la distribución de los centros de salud, se evidencia una mayor concentración y superposición de áreas de influencia en el sur y centro provincial. Los mapas de puntos de calor permiten evidenciar claramente la excesiva concentración de centros de salud en las principales aglomeraciones.

De la comparación de la tipología de centros, se desprende que la oferta privada de centros de salud compensa los déficits en la oferta pública, principalmente en Rosario, Santa Fe y Venado Tuerto. No obstante, es insuficiente en distritos medianamente poblados como Rafaela y Reconquista-Avellaneda (donde la mayoría de los centros son públicos). Los centros de salud privada compensan las necesidades de un sistema de salud pública saturado o colapsado para los casos de Rosario, Santa Fe y Venado Tuerto, haciendo disminuir la cantidad de habitantes por centros de salud en general. No resulta suficiente para los casos de Rafaela y Reconquista, cuyos valores de número de habitantes por centro de salud a atender superan los 3.000. La situación es notoriamente grave para el caso del aglomerado del norte santafesino, donde los valores de población con NBI son los más altos de la provincia.

En cuanto a la accesibilidad al servicio objeto de este estudio, como parámetro de la equidad social y la eficiencia espacial, se evidencian profundas disparidades: distritos donde se superponen ofertas (como en Rosario), mientras que en otros se carece o es deficiente (norte provincial). El mapa de la distribución de las distancias requeridas para llegar en tiempo a un centro de internación general da cuenta de los grandes desequilibrios provinciales que existen en materia de accesibilidad

Los servicios asistenciales, como la salud, deben ocupar un lugar central entre los objetivos de la planificación y ordenación territorial, para poder tender a la mejora de la calidad de vida de la sociedad en su conjunto. 


\section{Referencias bibliográficas}

Antúnez, I. y Galilea, S. (2003). Servicios públicos urbanos y gestión local en América Latina y el Caribe. Problemas, metodologías y políticas, Serie Medio Ambiente y Desarrollo, $\mathrm{N}^{\circ}$ 69. División Desarrollo Sostenible y Asentamientos Humanos. CEPAL.

Buzai G. D. y Baxendale C. A. (2006). Análisis socio espacial con sistemas de información geográfica, Buenos Aires: Lugar.

Comisión Económica para América Latina y el Caribe (CEPAL) (2019). Panorama Social de América Latina, 2018 LC/PUB.2019/3-P, Santiago.

Harvey, D. (1973). Social justice and the city, Madrid: Siglo XXI.

Instituto Nacional de Estadística y Censos (INDEC) (2010). Censo Nacional de Población, Hogares y Viviendas, 2010 (Datos definitivos).

Instituto Provincial de Estadística y Censo, Argentina. Censo Nacional de Población, Hogares y Viviendas (IPEC) (20I0). Censo Nacional de Población, Hogares y Viviendas. Escala de radios y fracciones censales.

Joseph A. y Phillips D. (1984). Accessibility \& Utilization. Geographical Perspectives on Health Care Delivery, New York: Harper \& Row.

Manzi, R. y Gallardo, M. (1970). Geografia de Santa Fe, Tomo I. Mendoza: Spadoni.

Ministerio de Salud (20I7). Provincia de Santa Fe. Informes 2017.

Moreno Jiménez, A. y Escolano Utrilla, S. (1992). Los servicios y el territorio, Madrid: Síntesis.

Moreno Jiménez, A. y Vinuesa Angulo, J. (2009). Desequilibrios y reequilibrios intrametropolitanos: principios de evaluación y metodología de análisis, Ciudad y territorio. Estudios territoriales, $\mathrm{N}^{\circ} \mathrm{I} 60$ (XLI), pp. $233-262$. Ministerio de Vivienda de España.

Savoy, F.; Carñel, G.; Curto, S. y Muller, G. (2012). Cobertura espacial bruta de la red provincial de hospitales de alta complejidad en Entre Ríos, Argentina, Contribuciones Cientificas GAA, Vol. 24, pp. 87-95. 